\title{
Cueva de la Cocina (Dos Aguas, Valencia) 2016: Intervención, METODOLOGÍA, RESULTADOS
}

ORETO GARCÍA PUCHOL ${ }^{1}$, AGUSTÍN DIEZ CASTILLO ${ }^{2}$, SARAH B. McCLURE ${ }^{3}$, JOAQUIM JUAN CABANILLES ${ }^{4}$, SALVADOR PARDO GORDÓ ${ }^{5}$, ALFREDO CORTELL NICOLAU ${ }^{5}$, PILAR ESCRIBÁ RUIZ ${ }^{5}$

(1) Grup d'Investigació PREMEDOC INV-GIUV-270642. Dpt. Prehistòria, Arqueologia i Història Antiga. Universitat de València. oreto.garcia@uv.es

(2) Grup d'Investigació GRAM. Dpt. Prehistòria, Arqueologia i Història Antiga. Universitat de València. adiez@uv.es

(3) Dpt. of Anthropology. The Pennsylvania State University.sbm19@psu.edu

(4) Museu de Prehistòria - Servei d’Investigació Prehistòrica. Diputació de València. Joaquim.Juan@dival.es

(5) Dpt. Prehistòria, Arqueologia i Història Antiga. Universitat de València.pargor@uv.es, alfredo.cortell@ext.uv.es, piesru2@gmail.com

\section{INTRODUCCIÓN}

En el verano de 2016, entre los meses de julio y septiembre, se ha llevado a cabo la segunda campaña de excavación en Cueva de la Cocina (Dos Aguas, Valencia) en el marco del programa de excavaciones arqueológicas del SIP-Museu de Prehistòria de València ${ }^{1}$. Los trabajos han estado guiados por los resultados de la campaña anterior, en la que pudimos realizar un total de seis sondeos en diferentes puntos de la cavidad situados en las inmediaciones de los sectores intervenidos por L. Pericot en los años 1940 (ver Pericot 1945) y J. Fortea en los años 1970 y 1980 (ver Fortea et al. 1987). La apertura de estos sondeos ha permitido aportar información pormenorizada desde las modernas técnicas de excavación actual, contribuyendo a la lectura de la interesante secuencia de la cavidad, que incluye una larga serie de ocupaciones atribuidas a los últimos cazadores-recolectores mesolíticos, al primer neolítico y a otras etapas prehistóricas posteriores hasta la Edad del Bronce (García Puchol et al. 2014a y b; 2015, e.p.; Pascual Benito y García Puchol 2015).

La Cueva de la Cocina se sitúa en un pronunciado meandro del denominado Barranco de la Ventana, que forma parte de la red de drenaje del valle de La Canal de Dos
Aguas, pequeño altiplano rodeado de elevaciones que se enmarcan en el sistema Ibérico, delimitado al $\mathrm{N}$ y el E con la llanura litoral valenciana y al sur con la salida del río Xúquer hacia el mar (fig. 1). La localización de la cavidad, en un entorno de media montaña con fácil acceso hacia los territorios costeros, justifica el interés puesto en los testimonios paeoambientales y económicos que contiene con el objetivo de contrastar las dinámicas socioecológicas de los grupos humanos ocupantes y, en especial, las implicaciones que se derivan respecto al desarrollo de los últimos caza-recolectores y la aparición de la economía de producción. Los trabajos actuales en cueva de la Cocina inciden de forma también especial en la obtención de datos estratigráficos que arrojen luz sobre las particularidades diacrónicas de la formación de los depósitos desde un punto de vista sedimentológico, ambiental y antrópico. Aspectos de los que adolecían en gran medida las intervenciones anteriores (Pericot 1945; Fortea 1971; Fortea 1973; Fortea et al. 1987). En los últimos años, y en el marco del proyecto HAR12-33111, se ha avanzado de forma sustancial en la información disponible mediante un programa multidisciplinar que incluye la reevaluación de la secuencia a través del análisis de los restos materiales y paleoeconómicos, y 


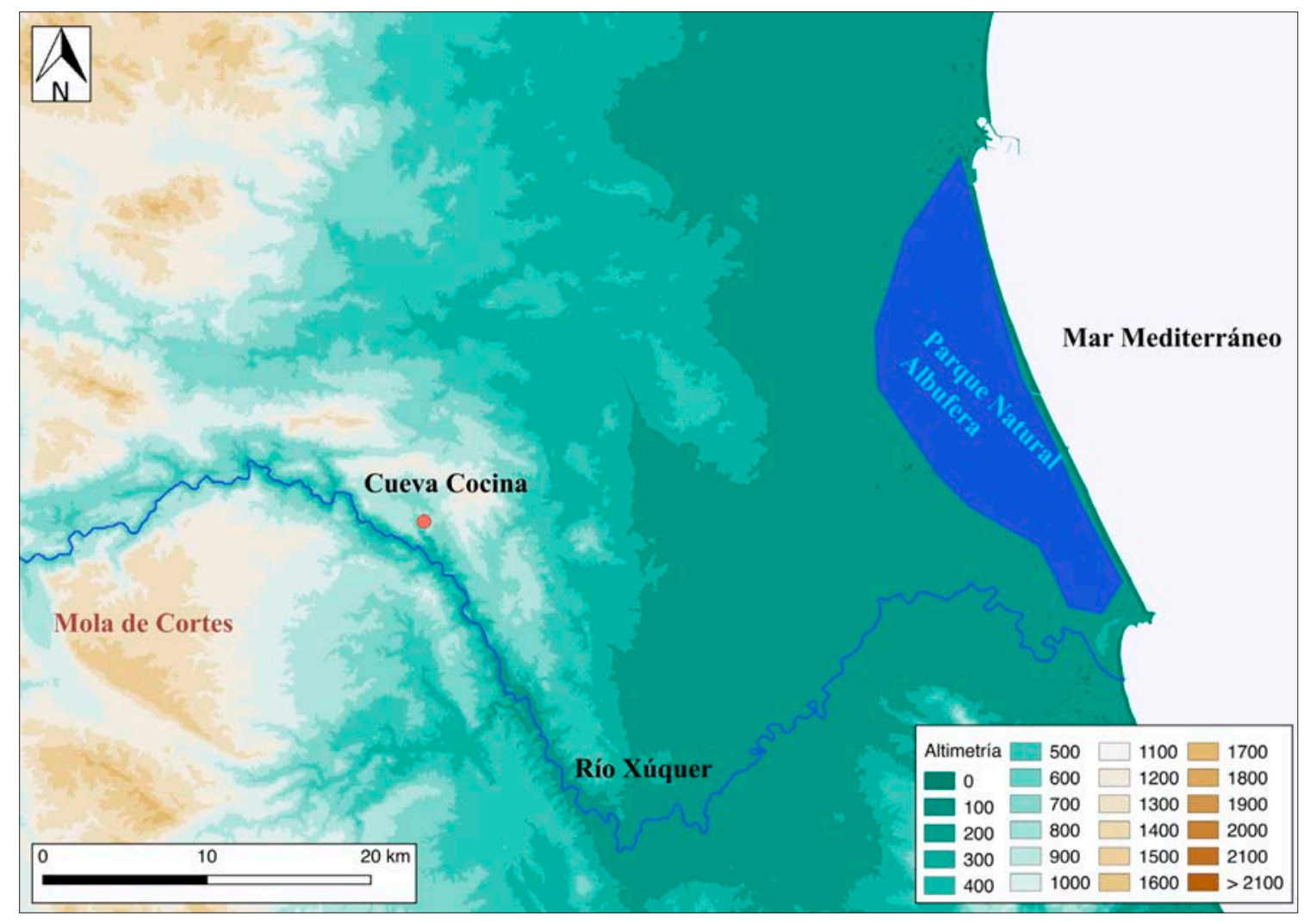

Fig. 1: Plano de situación de Cueva de la Cocina.

su referencia a un marco radiométrico cada vez más preciso (Juan Cabanilles y García Puchol 2013; García Puchol et al.e.p.).

\section{METODOLOGÍA}

La segunda intervención arqueológica en Cocina, descrita en esta nota, se ha centrado en uno de los nuevos sectores abiertos en la campaña anterior, el sondeo 4, dado que en él pudimos comprobar la existencia de depósitos mesolíticos, en apariencia intactos, en las inmediaciones de la gran cata excavada por Pericot en los años 1940 (fig. 2). El trabajo de esta campaña ha ido dirigido principalmente a ampliar el mencionado sondeo, situado cerca de la entrada de la cavidad y a avanzar también en profundidad tratando de reconocer las características del deposito arqueológico. Dada la información previa disponible sobre la aparición temprana del depósito natural de base hacia el interior de la cueva decidimos ampliar la cata hacia el exterior, de forma que si nos situamos en la planta de la cavidad, el sondeo practicado se localiza en una zona que debería enlazar con los sectores excavados por Pericot en los años 1943 y 1945 (sector EII).

La cata abierta se corresponde con los cuadros JE 1S, JE 2S, KE 1S, KE 2S, LE 1S (subcuadros 3 y 4) y LE 2 S. En total, la superficie que ocupa es de $5,5 \mathrm{~m}^{2}$, rebajados de forma desigual entre los diferentes cuadros, y alcanzando un máximo de profundidad de $50 \mathrm{~cm}$ en el cuadro LE $2 \mathrm{~S}$. El sistema de excavación ha seguido un riguroso registro de los hallazgos en planta una vez alcanzado el nivel arqueológico conservado. Se ha aplicado un protocolo digital (Diez et al. 2016) para la toma de datos que consiste en el registro de las fichas correspondientes a las UUEE mediante el uso del programa FileMaker, instalado en dispositivos equipados con el sistema operativo iOS. Además de la facilidad de uso que aporta FileMaker cabe destacar que se pueden realizar fotografías de cada una de las UUEE desde la propia aplicación, quedando así incorporadas a la ficha correspondiente. Hasta alcanzar los referidos $50 \mathrm{~cm}$ 


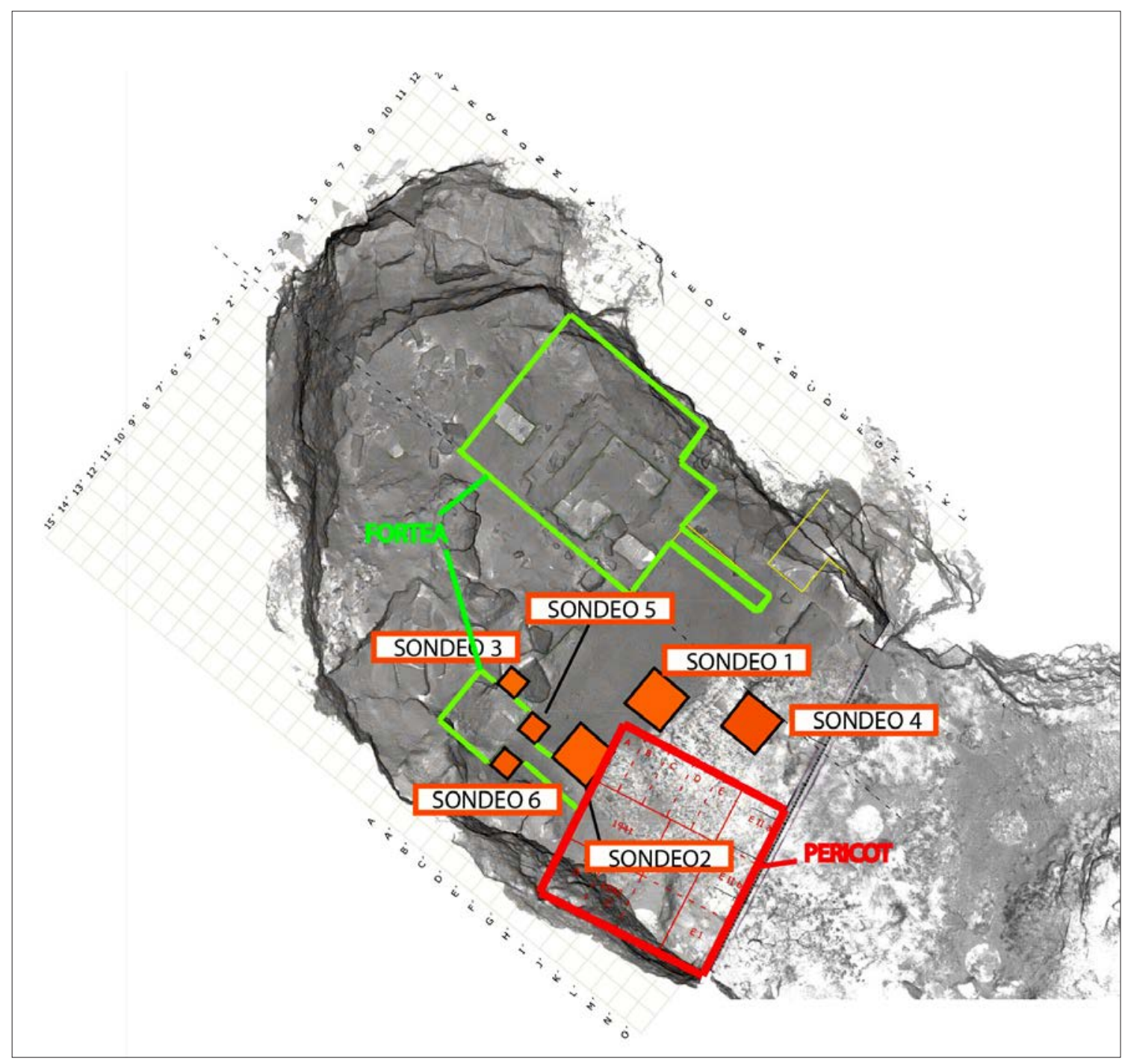

Fig. 2: Planta de la cavidad con indicación de los sectores excavados según las diferentes intervenciones efectuadas hasta la fecha. En rojo, intervenciones de L. Pericot; en verde, trabajos de Javier Fortea; en naranja excavaciones actuales.

se han rebajado UUEE de un máximo de $5 \mathrm{~cm}$, consignándose toda la información en las correspondientes fichas, toda vez que los materiales relevantes han sido registrados mediante coordenadas tridimensionales con el apoyo de una estación total Leica TCR-705. Estas coordenadas tridimensionales son descargadas en el laboratorio e incorporadas a las fichas de registro digitales.

Una vez alcanzado el nivel fértil, se ha seguido un meticuloso trabajo de registro de todos los materiales y excavado capas de un máximo de $2 \mathrm{~cm}$. Todo el sedimento ha sido recogido para su posterior lavado con agua en el laboratorio, donde se procede seguidamente a la separación de los diferentes tipos de materiales (arqueológicos y biológicos). Durante esta campaña hemos incorporado la fotogrametría en la labor de campo con el fin de obtener planos de plantas donde es posible realizar la medición exacta de sus componentes. Para ello, se han puesto en práctica modelos tridimensionales a partir de fotografías, con el apoyo de la aplicación PhotoScan de Agisoft (fig. 3). Una cámara Canon Eos1100D ha sido utilizada para realizar el registro fotográfico de las plantas requeridas. 


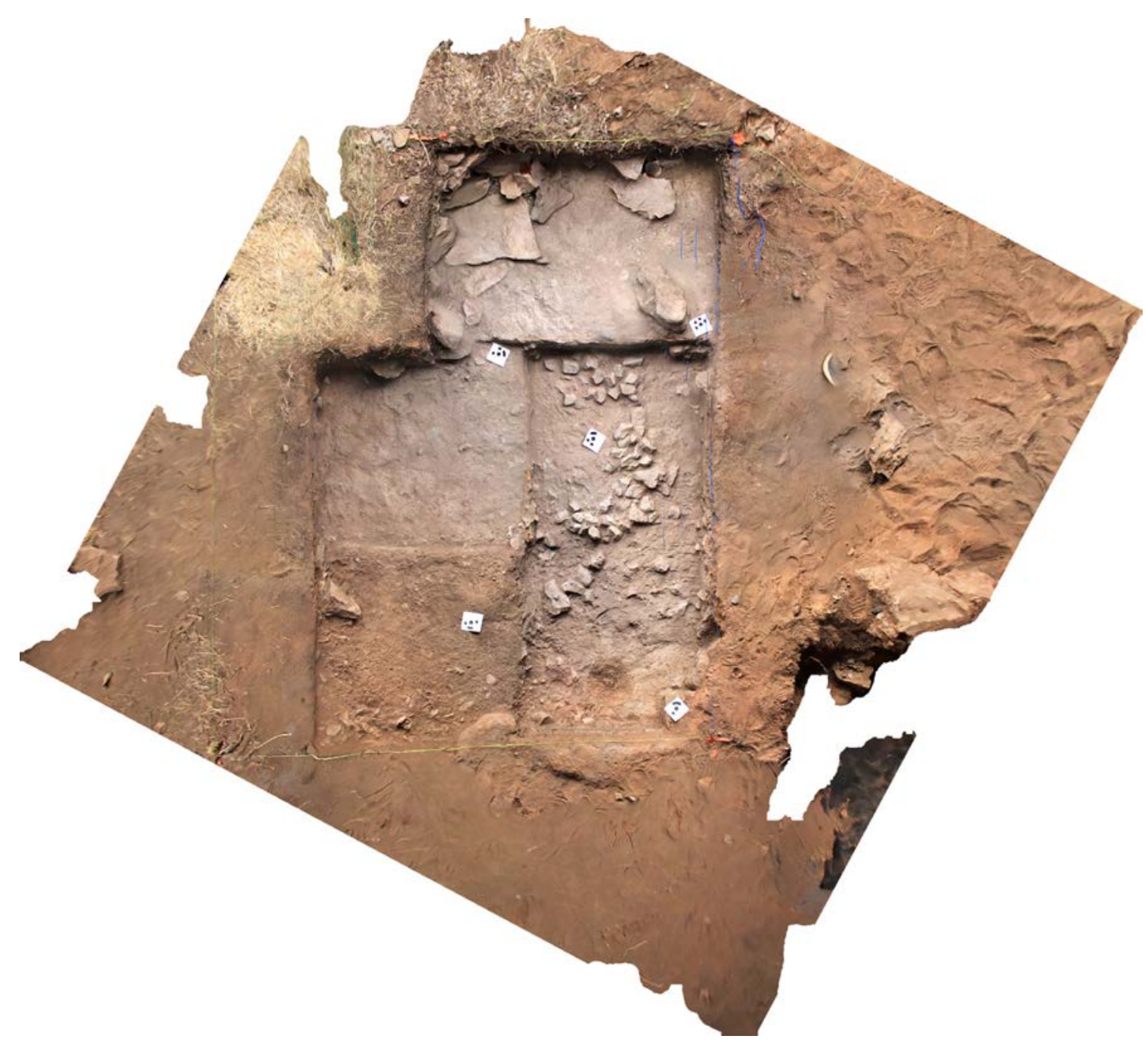

Fig. 3. Fotogrametría efectuada en el sondeo 4 al final de la intervención de 2016.

\section{RESULTADOS}

La figura 3 muestra la planta final de la intervención del sondeo 4, en la cual podemos ver el avance de la excavación en dos de los cuadros (JE 1S, KE 1S). Previamente habíamos procedido a ampliar la cata hacia los cuadros LE $1 \mathrm{~S}$ y LE $2 \mathrm{~S}$ con el fin de avanzar hacia el exterior de la cavidad y poder así comprobar la estratigrafía excavada por Pericot en el año 1945 (II). En este punto Pericot describió una bolsada estéril, además de la presencia de un paquete donde la cerámica prehistórica, en parte de adscripción neolítica, estaba presente. Por debajo refirió un nivel con materiales mesolíticos. La excavación superficial de los mencionados cuadros nos ha permitido observar la existencia de un paquete inicial con abundantes clastos, caídos de la cercana visera de la entrada, en el cual aparece cerámica moderna. Por debajo, un paquete limoso amarillento parece coincidir con la bolsada descrita por Pericot.

En los cuadros JE $1 \mathrm{~S}$ y JE $2 \mathrm{~S}$ se han rebajado aproximadamente unos $20 \mathrm{~cm}$ de depósito intacto que han proporcionado una importante cuantía de materiales prehistóricos, principalmente restos de talla lítica (abundantes laminitas) y algún geométrico, además de restos de fauna y malacofauna (terrestre y marina). El depósito conservado estaba protegido por la base del muro de cierre antiguo de 
la cueva que ya describió Pericot cuando excavó los sondeos adyacentes (Pericot 1945). Su presencia ha propiciado la preservación de depósito intacto en esta área de la cavidad. Los restos recuperados sugieren una atribución al Mesolítico Geométrico en una fase avanzada (con triángulos tipo Cocina). La extensión del depósito intacto hacia los cuadros adyacentes, y también en profundidad, podrá ser valorada adecuadamente en los futuros trabajos de campo.

\section{NOTA}

1. El trabajo presentado se ha llevado a cabo en el marco del proyecto HAR2015-68962 "EVOLPAST: Dinámicas evolutivas y patrones de variabilidad cultural de los últimos caza-recolectores y el primer neolítico en el Este peninsular (circa 70004500 cal BC)", Ministerio de Economía y Competitividad, Gobierno de España. Los trabajos de campo (Campaña 2016) se han realizado dentro del programa de investigaciones del SIP-Museu de Prehistòria, Diputación de Valencia.

\section{BIBLIOGRAFÍA}

FORTEA, J. (1971): La Cueva de la Cocina. Ensayo de cronología del Epipaleolítico (facies Geométricas), Valencia.

FORTEA, J. (1973): Los complejos microlaminares y geométricos del Epipaleolítico mediterráneo español, Salamanca

FORTEA, J.; MARTÍ, B.; FUMANAL, P. DUPRÉ, M.; PÉREZ RIPOLL, M. (1987): Epipaleolítico y neolitización en la zona oriental de la Península Ibérica, Premières Communautés Paysannes en Méditerranée Occidental. Actes du Colloque International du CNRS (J. Guilaine, J. Courtin, J.-L. Roudil, J.-L. Vernet, dir.), Paris, 599-606.

FUMANAL, M. P. (1986): Sedimentología y clima del País Valenciano: las cuevas habitadas en el Cuaternario reciente, Serie Trabajos Varios. S.I.P. 83. Valencia.

DIEZ, A.; CORTELL, A.; GARCÍA PUCHOL, O. (2016): Implementación de un SIG en la Cueva de la Cocina (Dos Aguas, Valencia), 10as Jornadas de SIG Libre, Universitat de Girona. Disponible en https://www.academia.edu/26741747/ Implementaci\%C3\%B3n_de_un_SIG_en_la_Cueva_de_ la_Cocina_Dos_Aguas_Valencia_[5/10/2016]

GARCÍA PUCHOL, O.; McCLURE, S. B., JUAN CABANILLES, J.; DIEZ, A.; BERNABEU, J.; MARTÍ, B.; PARDO GORDÓ, S.; PASCUAL BENITO, J. Ll.; PÉREZ RIPOLL, M.; MOLINA, L1.; KENNETT, D. J. (e.p.): Cocina Cave Revisited: Bayesian radiocarbon chronology for the last hunter-gatherers and first farmers in Eastern Iberia, Quaternary International.
GARCÍA PUCHOL, O.; JUAN CABANILLES, J.; McCLURE, S. B., DIEZ, A.; PARDO GORDÓ, S. (2015): Avance de resultados de los nuevos trabajos arqueológicos en Cueva de la Cocina (Dos Aguas, Valencia): campaña 2015, Sagvntvm-PLAV 47, 251-255. DOI: https://doi.org/10.7203/SAGVNTVM.47.7195

GARCÍA PUCHOL, O.; JUAN CABANILLES, J.; McCLURE, S. B.; PASCUAL BENITO, J. L1.; MARTÍ, B.; PÉREZ RIPOLL, M.; BERNABEU, J.; PARDO-GORDÓ, S.; MOLINA, L1.; CARRIÓN, Y.; DIEZ, A. (2014a): Los últimos caza-recolectores en Cueva de la Cocina (Dos Aguas, Valencia), Los cazadores y recolectores del Pleistoceno y del Holoceno en Iberia y el estrecho de Gibraltar (R. Sala, coord.), Universidad de Burgos/Fundación Atapuerca, $370-372$.

GARCÍA PUCHOL, O.; MAZZUCCO, N.; GIBAJA, J. F.; JUAN CABANILLES, J. (2014b): Use-wear analysis of a set of geometric projectiles from the Mesolithic context of Cocina Cave (Eastern Spain), International conference of use-wear analysis (J. Marreiros, N. Bicho, J. F. Gibaja, eds.), Newcastle upon Tyne, 213-224.

PASCUAL BENITO, J. Ll.; GARCÍA PUCHOL, O. (2015): Los moluscos marinos del Mesolítico de la Cueva de la Cocina (Dos Aguas, Valencia). Análisis arqueomalacológico de la campaña de 1941, La investigación arqueomalacológica en la Península Ibérica: nuevas aportaciones (I. Gutiérrez Zugasti, D. Cuenca, M. R. González Morales, eds.), Santander, 65-77.

PERICOT, L. (1945): La Cueva de la Cocina (Dos Aguas), APL II, 39-73. 